\title{
AMENDMENTS
}

\section{Author Correction: Instantaneous rock transformations in the deep crust driven by reactive fluid flow}

\author{
A. Beinlich (D), T. John (D), J. C. Vrijmoed, M. Tominaga (D), T. Magna and Y. Y. Podladchikov (D) \\ Correction to: Nature Geoscience https://doi.org/10.1038/s41561-020-0554-9, published online 16 March 2020.
}

In the version of this Article originally published, not all funding from Deutsche Forschungsgemeinschaft had been included. In the Acknowledgements, the sentence "The Deutsche Forschungsgemeinschaft financially supported this research through grant JO 349/5-1" has now been modified to read "The Deutsche Forschungsgemeinschaft (DFG) financially supported this research through grant JO 349/5-1 and grant CRC 1114 'Scaling Cascades in Complex Systems', Project Number 235221301, Project (C09) - 'Dynamics of rock dehydration on multiple scales"'

Published online: 22 December 2020

https://doi.org/10.1038/s41561-020-00683-Z

(c) The Author(s), under exclusive licence to Springer Nature Limited 2020

\section{Author Correction: Record of modern-style plate tectonics in the Palaeoproterozoic Trans-Hudson orogen}

O. M. Weller and M. R. St-Onge

Correction to: Nature Geoscience https://doi.org/10.1038/ngeo2904, published online 6 March 2017.

In the Supplementary Information file for this Article, in Supplementary Fig. 15, panel b was incorrect and wasn't of a mafic boudin within the Kovik tectonic window as specified in the caption and marked on the map in panel a. Because the correct image, a field photograph of this location taken in 1991, has been lost, panel b cannot be replaced and thus has been removed. Moreover, as panel d, the correlative field photograph from the Himalayan orogen, is only relevant if compared with panel b, it has also been removed. Accordingly, panel $c$ has been re-labelled as panel $b$ and the caption updated. We thank D. Corrigan (Geological Survey of Canada) for alerting us to the error. Furthermore, in the main article, in the sentence beginning "The congruence is particularly striking..." "(Supplementary Fig. 15a,c)" should thus be "(Supplementary Fig. 15a,b)", and "(Supplementary Fig. 15b,d)" is not relevant. The amended Supplementary Information file is supplied with this correction notice. The original Article has not been corrected.

\section{Additional information}

Supplementary Information The online version contains supplementary material available at https://doi.org/10.1038/s41561-020-00685-x.

Published online: 24 December 2020

https://doi.org/10.1038/s41561-020-00685-X

(c) The Author(s), under exclusive licence to Springer Nature Limited 2020 